BULLETIN OF THE

AMERICAN MATHEMATICAL SOCIETY

Volume 79, Number 4, July 1973

\title{
GROTHENDIECK AND WHITEHEAD GROUPS OF TORSION FREE ABELIAN GROUPS
}

\author{
BY DAVID M. ARNOLD \\ Communicated by Joseph Rotman, December 19, 1972
}

Let $\mathscr{A}$ denote the category of torsion free abelian groups of finite rank and let $K_{0}(\mathscr{A})$ and $K^{0}(\mathscr{A})$ be the Grothendieck groups of $\mathscr{A}$ (modulo split exact sequences and exact sequences, respectively).

J. Rotman [5] determined the group and ring structure of $K^{0}(\mathscr{A})$; in particular, $K^{0}(\mathscr{A})$ is a free abelian group of uncountable rank. There is a canonical epimorphism $\pi_{0}: K_{0}(\mathscr{A}) \rightarrow K^{0}(\mathscr{A})$ so $K_{0}(\mathscr{A})$ has a free summand.

Let $\mathscr{C}$ be the full subcategory of $\mathscr{A}$ consisting of groups with constant $p$-rank (i.e., there is an integer $n$ such that the $Z / p Z$-dimension of $A / p A$ is equal to $n$ for all primes $p$ ). Define $K_{0}(\mathscr{C})$ to be the Grothendieck group of $\mathscr{C}$ (modulo split exact sequences) and let $\tilde{K}_{0}(\mathscr{C})$ be the kernel of the rank homomorphism from $K_{0}(\mathscr{C})$ to $Z$, the ring of integers.

Proposition 1. $\widetilde{K}_{0}(\mathscr{C})$ is isomorphic to the kernel of $\pi_{0}$.

The category $\mathscr{C}^{\prime}$ is defined by letting the objects of $\mathscr{C}^{\prime}$ be the objects of $\mathscr{C}$, and with morphism sets $Q \otimes_{\mathrm{Z}} \operatorname{Hom}_{\mathrm{Z}}(A, B)$ for groups $A$ and $B$ in $\mathscr{C}^{\prime}$. There is a canonical epimorphism $\sigma_{0}: K_{0}(\mathscr{C}) \rightarrow K_{0}\left(\mathscr{C}^{\prime}\right)$. Moreover, $K_{0}\left(\mathscr{C}^{\prime}\right)$ is a free abelian group (of uncountable rank) since $\mathscr{C}^{\prime}$ has a KrullSchmidt theorem (e.g., see Walker [6]).

If $R$ is a ring with identity, then $K_{0}(R)$ is defined to be $K_{0}\left(\mathscr{P}_{R}\right)=K^{0}\left(\mathscr{P}_{R}\right)$, $\mathscr{P}_{R}$ the category of finitely generated projective $R$-modules.

A corollary to the next theorem is: The torsion subgroup of $K_{0}(\mathscr{A})$ is nonzero (for $K_{0}(R) \simeq Z \oplus I(R)$, where $I(R)$ is the ideal class group of $R)$.

THeOREM 2. Suppose that $R$ is a Dedekind domain such that $R^{+}$, the additive group of $R$, is a reduced torsion free abelian group of finite rank. Then $K_{0}(R)$ is isomorphic to a subgroup of $K_{0}(\mathscr{A})$.

Let $K_{1}(\mathscr{A})$ be the Whitehead group of $\mathscr{A}$ (as defined by Bass [3]). Since $\mathscr{C}$ is a cofinal subcategory of $\mathscr{A}$, we have

Corollary 3. $K_{1}(\mathscr{C}) \simeq K_{1}(\mathscr{A})$.

AMS (MOS) subject classifications (1970). Primary 20K15, 18F25; Secondary 13F05, $13 \mathrm{D} 15$.

Key words and phrases. Torsion free abelian groups of finite rank, Grothendieck groups, Whitehead groups. 
Define $\mathscr{P}(\mathscr{C})$ to be the collection of groups in $\mathscr{C}$ with constant $p$-rank 1 and with product $A * B=\left(A \otimes_{Z} B\right) / d\left(A \otimes_{Z} B\right)$, where $d\left(A \otimes_{Z} B\right)$ is the divisible subgroup of $A \otimes_{Z} B$. Let $J$ be the product, over all primes $p$, of the $p$-adic integers; $U(J)$ the multiplicative group of units in $J$; and $A U(J)$ the group of algebraic units of $J$, i.e., $A U(J)=\{x \in U(J) \mid f(x)=0$ for some nonzero polynomial $f$ with integral coefficients $\}$.

THEOREM 4. (a) There are group epimorphisms $d_{i}: K_{i}(\mathscr{C}) \rightarrow K_{i}(\mathscr{P}(\mathscr{C}))$ for $i=0,1$.

(b) $K_{1}(\mathscr{P}(\mathscr{C})) \simeq A U(J)$.

(c) $K_{1}\left(\mathscr{P}\left(\mathscr{C}^{\prime}\right)\right) \simeq A U(J) \oplus U^{+}(Q)$, where $U^{+}(Q)$ is the multiplicative group of positive rational numbers.

Let $H: \mathscr{C} \rightarrow \mathscr{P}_{J}$ be the ( $Z$-adic) completion functor. Then there is a commutative diagram

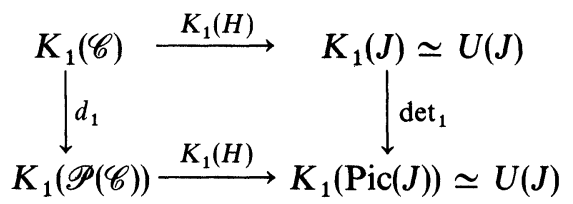

where $\operatorname{det}_{1}$ is the usual determinant homomorphism. Furthermore, $K_{1}(H)$ is a monomorphism on $K_{1}(\mathscr{P}(\mathscr{C}))$.

The structure of $K_{0}(\mathscr{P}(\mathscr{C}))$ remains an open question. Some partial results are contained in [2]; in particular, $K_{0}(\mathscr{P}(\mathscr{C}))$ has a summand isomorphic to $\Pi / \sum$, where $\Pi$ and $\sum$ are the direct product and sum, respectively, of a countable number of copies of $Z$.

\section{REFERENCES}

1. D. M. Arnold and E. L. Lady, Endomorphism rings of direct sums of torsion free abelian groups, (to appear).

2. D. M. Arnold, Algebraic K-theory and torsion free abelian groups of finite rank, Symposia Mathematica, Academic Press, New York, (to appear).

3. H. Bass, Algebraic K-theory, Benjamin, New York, 1968. MR 40 \#2736.

4. J. Milnor, Introduction to algebraic K-theory, Annals of Math. Studies 72 , Princeton Univ. Press, 1971.

5. J. Rotman, The Grothendieck group of torsion free abelian groups of finite rank, Proc. London Math. Soc. (3) XIII (1963), 724-732. MR 27 \# 4857.

6. E. Walker, Quotient categories and quasi-isomorphism of abelian groups, Proceedings of Colloquium on Abelian Groups, Budapest, 1964, 147-162. MR 31 \#2327.

Department of Mathematical Sciences, New Mexico State University, Las Cruces, New MEXICO 88003 